\title{
Study on performance and flow field simulation of a new type precipitator
}

\author{
Junyi Yin ${ }^{1, a}$ \\ ${ }^{1}$ North China Electric Power University, Baoding 071003, China; \\ a2807129564@qq.com
}

Keywords: Composite precipitator, dedusting Mechanism, dedusting efficiency, Numerical Simulation

\begin{abstract}
We design a new type of composite precipitator model which is an organic combination of the mechanism of the Electrostatic precipitator of Tubular Electrostatic Precipitator, the Centrifugal dedusting of Cyclone type ESP, and cleaning water film. We use the outer cylinder of cyclone precipitator as the dust pole of tubular electrostatic precipitator, the flue gas discharge tube of cyclone precipitator as discharge electrode. We take hollow prick epipolar line into practice, with circular tube in the middle of it used as flue gas outlet, and there are pricks welded around it. We set up waterfall devices on the top of the dust pole, by doing simulation and comprehensive testing of its electric field and flow field, it is obvious that the new type precipitator we designed has an ideal efficiency of dedusting flue particulates such as PM2.5.
\end{abstract}

\section{Introduction}

Research shows that the main causes of fog and haze are the emission of industrial flue gas particulates. With the rapid expansion of economies scale and accelerating urbanization in recent years, Atmospheric aerosol pollution is increasingly serious, especially the micron and submicron level fine particles whose particle size less than $10 \mu \mathrm{m}$, which is harm to human health seriously. And for air purify or for industrial dust, its particle size is too small and difficult to remove. Therefore, purification processing of fine particulate is a hot issue all over the world [1].

Currently, the commonly used dedusting equipments in industry include cyclone dust, ESP and so on. Conventional cyclone or multi-tube cyclone dust stream is to rotary motion by means of centrifugal force to separate and trap dust particles from the air stream to the wall, and then with the force of gravity so that dust particles fall into the hopper, collection efficiency is generally $75 \%$ to $85 \%$, and for the poor performance to adapt to changes in load, it is difficult to meet increasingly stringent environmental requirements. Conventional electrostatic precipitator by applying a high voltage between the poles asymmetrical cathanode, so that the surrounding gas ionizing electrode, thereby enabling the charged particles in the flue gas, charged particles in the electric field strength anisotropy electrode movement, and is trapped in the plates. Final cleaning through the electrode, so that dust falls into the hopper, so to achieve the clear purpose of Flue gas dust, but are mostly used ESP rapping cleaning methods can easily lead to a secondary dust. Wet electrostatic precipitator typically by the way of waterfall water to rinse off the dust deposited on the surface of the dust collecting plate[2], but problems such as maldistribution of water film flow will arise in the process of decline, leading to the surface of the dust collecting plate some dust areas can not be cleared, or appear dry spots and ash hardening, can easily cause back corona phenomenon, lower collection efficiency. What's more, to avoid dry spots and ash hardening, we need to increase the amount of water for flushing, which resulted in a waste of water resources[3].

In this paper, the design of a new composite precipitator, through the rational transformation of existing precipitators to form a high-voltage electrostatic field in the original cyclone, so that in the dual role of the centrifugal force and the electric field can be more effectively collect dust particles, especially fine dust, and through using wet cleaning method avoids the rapping of secondary dust cleaning. 


\section{The building of physical model}

The new type of composite precipitator model organically combines the mechanism of tubular electrostatic precipitator, Cyclone type ESP, and cleaning water film, includes the electrode assembly of tubular electrostatic precipitator, the air moving device cyclone ESP and uniform water film device. We use the outer cylinder of cyclone precipitator as the dust pole of tubular electrostatic precipitator, the flue gas discharge tube of cyclone precipitator as discharge electrode[4]. We take hollow prick epipolar line into practice, with circular tube in the middle of it used as flue gas outlet, and there are pricks welded around it. As is shown in Figure 1, there are three parts of dust collection plate, the upper section is the inlet section, a cylindrical structure; the middle is the transition section, the cone with a small angle tilt; the lower section is the hopper cone. At the top of the dust collector plate we set up waterfall device, it makes the water flowing from the first part of dust along the inside wall plate to the bottom plate of the dust, and by quenching the uniform water, adding an amount of surface active agent into water, so as to improve the collection efficiency of aerosol substances.

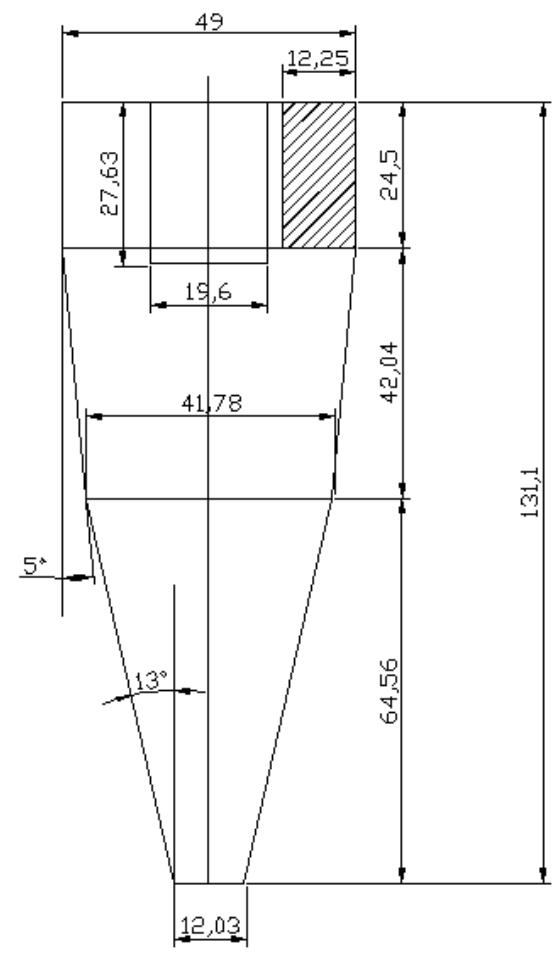

Fig. 1 The size of a new type of composite precipitator

\section{Two-phase FIELD VALUE IN analysis of composite precipitator}

Movement rule of the new type composite precipitator inside is a three-dimensional strong rotating turbulent flow field, but the distribution of the main features of characteristic quantities will change. We use FLUENT simulation software to simulate this new composite precipitator internal flow field and ultimately reach to the best size and angle to let this composite precipitator working in top condition.

The flow field simulation results show that best inlet air speed is $18.5 \mathrm{~m} / \mathrm{s}$, Flow rate is $2000 \mathrm{~m}^{3} / \mathrm{h}$. Under these conditions, we have stimulated the dedusting effect of different sizes fine particles, as shown in Figure 2-4, we find that it has a better effect on fine particles such as PM2.5, and this is one of the feature of our project. At the same time, the simulation of two-phase flow inside the precipitator and distribution (Figure 5), also received a relatively ideal result. Thus, this new composite precipitator we have envisaged has sufficient theoretical and practical feasibility. 


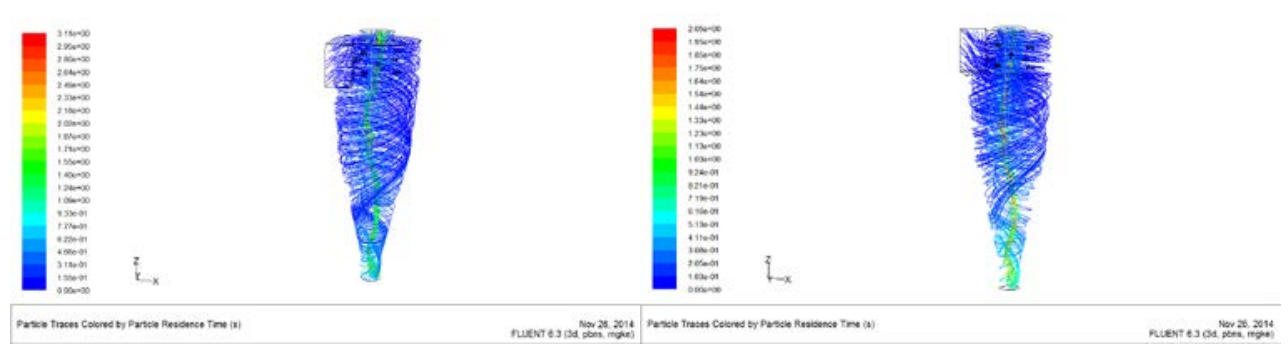

Fig. 2 The particulate trajectories of PM10 Fig. 3 The particulate trajectories of PM2.5

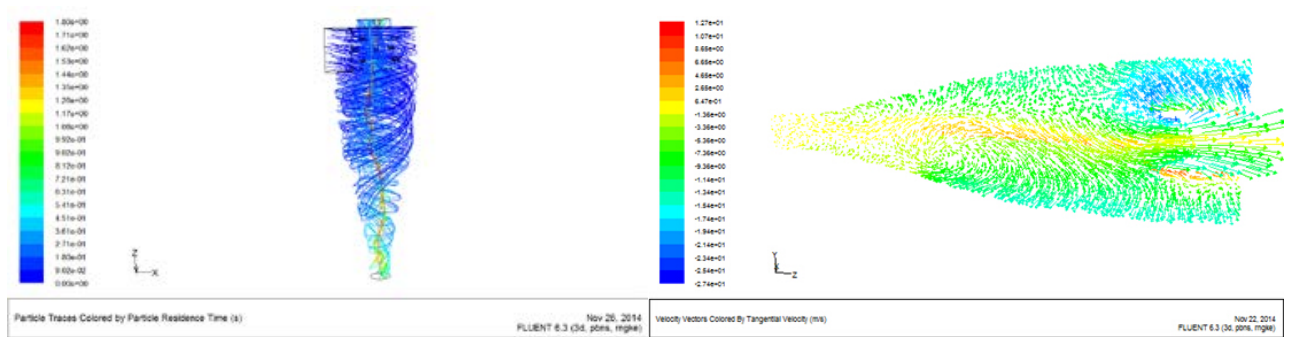

Fig. 4. The particulate trajectories of PM1 Fig. 5. Two-phase flow inside precipitator

\section{Performance Analysis of Composite Precipitator}

Stokes force, centrifugal force and the electrostatic force is essential in the separation process of the new composite precipitator, Stokes force present outside various separation processes, while the effects of centrifugal force and electrostatic precipitator is based on the specific structure and electrode arrangement position. The mechanisms are as follows:

Applying a DC voltage high enough cyclone between the electrostatic corona electrode and outer cylinder (dust pole), under the action of a very strong electric field between the corona pole and dust pole, the gas near the surface of the corona pole occurs ionization, appearing corona discharge and producing large amounts of positive ions and free electrons.

Most of the positive ions are attracted in the vicinity of the corona pole, while free electrons head toward the corona pole in the electric field strength, and thus the entire electric space filled with it. When the rotated dust stream into the electric field region, dust particles may be have a direct collision with free electrons; free electrons may also be absorbed by gas molecules and produce negative ions, in addition ash particles may have collided with it and charged. Therefore, when ash particles rotate with the air in the region of the electrostatic cyclone electric field, it is forced by centrifugal force Fc, electric force Fe, gas resistance and gravity. Ash particles with the joint effect of the centrifugal force and electric force is gradually being left out of the barrel wall , and under the action of gravity it rotates along the wall and fall into the hopper. What's more, waterfall water devices set up on the top of the dust pole avoid secondary dust effectively.

\section{Analysis of the water film cleaning effect}

Using three-stage structure of the dust collecting plate (Fig. 6) and the cyclone air, so that distribution of the water film will be more evenly, and the effect of capturing the fine particles is better. The reason is that: due to the force of gravity $\mathrm{G}$, water droplets which is in an inner wall with a certain tilt angle, has two components: one perpendicular to the plate surface so that the water attached to the wall panels, the other in an oblique direction makes the water flows along the plate down. Meanwhile, the presence of cyclonic airflow avoids the situation that the water film flow bifurcation and uneven effectively, so that the effect of cleaning is optimized. 


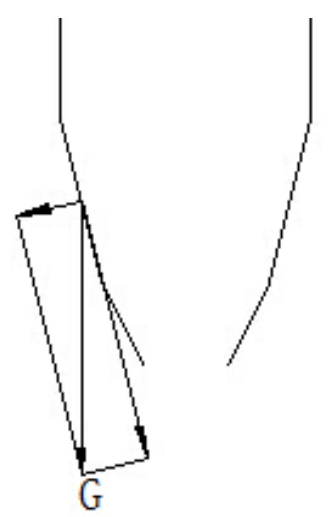

Fig. 6 the stress analysis of three-stage structure

\section{Conclusion}

To establish a physical model of a new type of composite precipitator aiming at the current problems in various precipitator, using FLUENT software simulation repeated its operation, and to find the optimum working status of each components dimensions and angles. Meanwhile, we through theoretical analysis and flow field, electric field simulation to verify the practical feasibility of this new type of composite precipitator.

The overall performance of this new composite precipitator better than conventional electric precipitator, with an excellent absorption efficiency of fine particles in industrial flue gas, it can reduce the secondary dust, anti-corona, the problem of uneven film of water effectively. What's more, it owns an ideal the absorption effect of PM2.5 and other tiny particles, expecting to ease the moment fog and haze.

Currently, the increasingly serious energy crisis and environmental issues make precipitators develop in an efficient direction; this composite precipitator is expected to have further development in the area of dealing with dealing with the gas which has low dust content and smaller particle size.

\section{References}

[1] Lifeng Yang. Jinhai Wang. Experimental study of effects of electrostatic corona electrode in the form of Cyclone dust Performance [J]. Energy Research and Information, 2006,22(2):63-68.

[2] Weijun. Li. The theoretical analysis of mechanism and efficiency of composite electrical cyclone dust [J]. Shanghai Polytechnic University, Feb. 2007.

[3] Jiguang Zhang. Hengyin Shen. Electrostatic cyclone flow field analysis [J] Thermal Power Engineering, 2002, 17 (101): 499-501.

[4] Jiguang Zhang. Daolian Wang. Experimental study of electrostatic cyclone flow field [J] Fluid Machinery, 2002, 30 (10): 4-7. 